\title{
RESEARCH
}

Open Access

\section{Volume and accumulation patterns of physical activity and sedentary time: longitudinal changes and tracking from early to late childhood}

Katherine L Downing ${ }^{1 *}$, Trina Hinkley ${ }^{1}$, Anna Timperio ${ }^{1}$, Jo Salmon ${ }^{1}$, Alison Carver ${ }^{2}$, Dylan P Cliff ${ }^{3}$, Anthony D Okely ${ }^{3}$ and Kylie D Hesketh ${ }^{1}$

\begin{abstract}
Background: Physical activity (PA) decreases and sedentary time (SED) increases across childhood, with both behaviours tracking. However, no studies have examined how accumulation patterns of PA and SED (i.e., prolonged bouts, frequency of breaks in sedentary time) change and track over time. The aim of this study was to investigate longitudinal changes in and tracking of total volume and accumulation patterns of SED, light-intensity PA (LPA), moderate-intensity PA (MPA), vigorous-intensity PA (VPA) among boys and girls.

Methods: In 2008/09 (T1), children in HAPPY (3-5y; $n=758$ ) in Melbourne, Australia wore ActiGraph GT1M accelerometers to objectively assess SED, LPA, MPA and VPA. This was repeated at age 6-8y (T2; $n=473)$ and 9-11y (T3; $n=478)$. Ten pattern variables were computed: bouts of $\geq 5-, \geq 10-, \geq 15$ - and $\geq 20$ - $m$ in for SED, $\geq 1$ - and $\geq 5$ min for LPA, $\geq 1$-min for MPA, $\geq 1$ - and $\geq 5$-min for VPA, and breaks in SED (interruptions of $>25$ counts $15 \mathrm{~s}^{-1}$ ). Longitudinal mixed models examined changes from T1-3, controlling for T1 age. Generalized estimating equations assessed tracking over the three time points, controlling for $\mathrm{T} 1$ age and time between measurements. Analyses were stratified by sex.

Results: Total volume and bouts of SED and SED breaks increased, while total volume and bouts of LPA decreased for both sexes. There was a small decrease in total volume of MPA for girls, but time spent in $\geq 1$-min bouts increased for both sexes. Total volume of VPA increased for both sexes, with time spent in $\geq 1$-min bouts increasing for boys only. All volume and pattern variables tracked moderately for boys, except for all SED bouts $\geq 15$-min, LPA bouts $\geq 5$-min and MPA bouts $\geq 1$-min (which tracked weakly). For girls, total SED and SED bouts $\geq 1$-min tracked strongly, total volume of LPA, MPA and VPA, $\geq 5$ - and $\geq 10$-min SED bouts, and $\geq 1$-min LPA and MPA bouts tracked moderately, and SED breaks, all SED bouts $\geq 15 \mathrm{~min}$, LPA bouts $\geq 5$ min and all VPA bouts tracked weakly.

(Continued on next page)
\end{abstract}

\footnotetext{
* Correspondence: k.downing@deakin.edu.au

${ }^{1}$ Institute for Physical Activity and Nutrition (IPAN), School of Exercise and

Nutrition Sciences, Deakin University, Geelong, Australia

Full list of author information is available at the end of the article
}

(c) The Author(s). 2021 Open Access This article is licensed under a Creative Commons Attribution 4.0 International License, which permits use, sharing, adaptation, distribution and reproduction in any medium or format, as long as you give appropriate credit to the original author(s) and the source, provide a link to the Creative Commons licence, and indicate if changes were made. The images or other third party material in this article are included in the article's Creative Commons licence, unless indicated otherwise in a credit line to the material. If material is not included in the article's Creative Commons licence and your intended use is not permitted by statutory regulation or exceeds the permitted use, you will need to obtain permission directly from the copyright holder. To view a copy of this licence, visit http://creativecommons.org/licenses/by/4.0/ The Creative Commons Public Domain Dedication waiver (http://creativecommons.org/publicdomain/zero/1.0/) applies to the data made available in this article, unless otherwise stated in a credit line to the data. 
(Continued from previous page)

Conclusions: Patterns of SED and PA change from early to late childhood; with the exception of SED breaks and VPA, changes were detrimental. Total volumes and short bouts tended to track more strongly than longer bouts. Interventions to prevent declines in PA and increases in SED are important from early in life.

Keywords: Sedentary behaviour, Physical activity, Stability, Longitudinal, Preschool, School-aged

\section{Introduction}

Engaging in sufficient physical activity and minimising time spent sedentary contribute to healthy development during and beyond early childhood $[1,2]$. In addition to total volume, accumulation patterns of physical activity and sedentary time (i.e., the number and duration of bouts and breaks in sedentary time) may be important for children's health. Although the benefits of reducing sedentary bouts are yet to be established in young children, breaking up prolonged bouts of sedentary time has been shown to be associated with more favourable cardio-metabolic profiles in school-aged children [3-5].

Although Australian preschool children (aged 3- to 5years) spend around 5-6 h per day being physically active $[6,7]$, with the majority meeting the physical activity guidelines of $180 \mathrm{~min} /$ day $[6,8]$, their physical activity is sporadic in nature [7]. In addition, they spend more than $50 \%$ of their waking hours sedentary [7]. Further, international cross-sectional data $(n>27,000)$ indicate that from age 5 years, when preschool children begin their transition to primary (elementary) school, physical activity starts to decline rapidly and sedentary time starts to increase, with boys more active and less sedentary than girls at all ages [9].

In addition to determining the nature of changes in physical activity and sedentary time, it is important to examine how these behaviours track. "Tracking" refers to the stability of a particular behaviour over time [10], or the maintenance of an individuals' relative rank over time within a cohort [11]. Jones et al. [12] conducted a systematic review of studies reporting tracking of physical activity and sedentary behaviours from early childhood. That review found evidence of moderate (coefficient 0.30-0.49) tracking of physical activity, and moderate to strong (coefficient $\geq 0.50$ ) tracking of sedentary behaviour (mostly in the form of sedentary screen time), during early childhood and from early childhood to middle childhood. However, just two of the 14 studies included in that review reported tracking of objectively assessed sedentary time, and six of the 14 studies reported tracking of objectively-assessed physical activity.

Studies published since that review that used accelerometers have reported similar results. Carson et al. [13] found moderate tracking for light-intensity physical activity (LPA), MVPA and sedentary time over three time points during early childhood (from age 1.6 to 3.7 years).
Caldwell et al. [14] found moderate tracking for LPA, moderate-intensity physical activity (MPA) and vigorous-intensity physical activity (VPA), as well as strong tracking for sedentary time over 1 year (age 4.5 to 5.5 years). Carson et al. [15] investigated tracking of sedentary time and bouts over a 1 year period among 3 - to 5 -year-olds, and found moderate tracking of total sedentary time, 1-4 min sedentary bouts, and 5-9 min sedentary bouts, but no tracking of $\geq 10$ min sedentary bouts. No studies have investigated tracking in boys and girls separately and no studies have investigated tracking of total volumes and accumulation patterns (bouts) of objectively measured physical activity and sedentary time over multiple time points. Identifying whether specific patterns of accumulation of sedentary time and physical activity track may help inform future intervention strategies. For example, if children's engagement in long bouts of sedentary time track moderately to strongly, future interventions could include approaches to break up prolonged sitting from a young age. The aim of this study was to investigate longitudinal changes and tracking of total volumes and patterns of accumulation of sedentary time, LPA, MPA and VPA from preschool (35 years) to late primary school (9-11 years) among boys and girls.

\section{Methods}

\section{Recruitment and participants}

Data for this study were from the Healthy Active Preschool and Primary Years (HAPPY) cohort study. Recruitment details for this study have been previously published [16]. Briefly, parents and their children were recruited from preschools and childcare centres in August-December 2008 and June-November 2009 in Melbourne, Australia. All parents with children aged 35 years attending participating centres were invited to participate, with 1002 parents/guardians providing consent at baseline (T1).

Although initially designed as a cross-sectional study, 766 parents $(77 \%)$ provided consent to be re-contacted for future research. These families were invited to participate in follow-up studies at three years post-baseline (from August 2011-March 2012 and June 2012-April 2013; T2) and at six years post-baseline (from July 2014May 2015 and June 2015-May 2016; T3). A total of 567 (74\%) and $568(76 \%)$ parents consented to T2 and T3, 
respectively. Deakin University Human Research Ethics Committee (EC291-2007), Department of Education and Early Childhood Development (2011_001008), and Catholic Education Melbourne (GE11/0009) approved the study. Parents provided written informed consent and children provided verbal assent to wear the accelerometers at each time point.

\section{Measures and data management}

At T1, parents reported their child's sex and date of birth (used to calculate age). Children were visited at preschool at T1, and at T2 and T3 parents opted for their child to be visited either at home or school, with data collected during school terms. Children were fitted with an ActiGraph GT1M uniaxial accelerometer (Pensacola, FL, USA) on an elastic belt at the right iliac crest. They were instructed to wear it during waking hours for eight consecutive days, only removing for water-based activities (e.g., bathing, swimming). Data were collected in 15-second epochs $[17,18]$ and were processed using a customised Microsoft Excel macro. Non-wear time was determined as $\geq 20$ min of consecutive zero counts [17, 19]. Children were required to have data recorded for $\geq$ $6 \mathrm{~h}$ per day (at T1) and $\geq 8 \mathrm{~h}$ per day (at T2 and T3) on $\geq 4$ days (including $\geq 1$ weekend day) $[20,21]$ to be included in the analyses. The lower wear time criterion used at T1 compared to T2 and T3 is specific to preschool children and accounts for their longer sleep time and subsequent shorter wake time (i.e., less time available for accelerometer wear time), compared with school-aged children. Sedentary time (SED) and physical activity were defined using cut points established by Evenson et al. [22](SED $\leq 25$, LPA 26-573, MPA 5741002 , and VPA $\geq 1003$ counts $15 \mathrm{~s}^{-1}$ ), which have been validated in preschool [23] and school-aged [24] children.

Patterns of accumulation were defined a-priori as bouts of $\geq 5$ - $\mathrm{min}, \geq 10-\mathrm{min}, \geq 15-\mathrm{min}$ and $\geq 20$-min of SED and $\geq 1$-min, $\geq 5$ - min and $\geq 10$-min of LPA, MPA, and VPA; however, only bout lengths that were recorded by $\geq 25 \%$ of the sample were included in analyses. Hence, bouts included in analyses were $\geq 5$ - $\mathrm{min}, \geq 10$ min, $\geq 15$-min and $\geq 20$ - $\min$ SED, $\geq 1$ - $\min$ and $\geq 5$-min LPA, $\geq 1$-min MPA, and $\geq 1-\mathrm{min}$ and $\geq 5$-min VPA bouts. Previous research has found that allowing exceptions (i.e., interruptions in intensity) increases the accumulation of time in longer bouts [25]; hence, no exceptions were allowed in this study. In addition, interruptions in SED time were defined as exceeding 25 counts $15 \mathrm{~s}^{-1}$ [26]. Mean values for all accelerometry variables were calculated over all valid days; for bouts, mean values were calculated including only children who participated in those bout lengths (i.e., if a child did not engage in a particular bout length it was treated as missing) to reduce potential tracking of zeroes. All accelerometer variables were standardised using the residuals obtained when regressing on wear time [27].

\section{Data analysis}

All analyses were conducted using Stata/SE 15.0 (StataCorp, Texas, USA) and were stratified by sex of the child. Descriptive analyses were used to summarise the data at each time point. Linear mixed models were used to determine change in each of the accelerometry variables (total time and bouts in each intensity [SED, LPA, MPA, VPA] and SED breaks), controlling for child's age at T1. Mixed-models fit a linear trend line for each child and do not require data at each time point, maximising the use of available data. Tracking of each of the accelerometry variables were examined using General Estimating Equations (GEE), which use all available data to calculate a single stability coefficient. As per previous tracking studies [28, 29], GEE were used to predict the value of each variable at time $t$ from the corresponding value at time $\mathrm{t}-1$, controlling for age at $\mathrm{T} 1$ and time between measurements. Tracking coefficients were standardised by applying the formula:

$$
\beta \mathrm{s}=\beta\left(\frac{\mathrm{sd}(\mathrm{Yt}-1)}{\mathrm{sd}(\mathrm{Yt})}\right)
$$

where $\beta_{\mathrm{s}}$ is the standardised tracking coefficient, $\beta$ is the non-standardised tracking coefficient, $\operatorname{sd}\left(\mathrm{Y}_{t}-1\right)$ and $\operatorname{sd}\left(\mathrm{Y}_{t}\right)$ are the standard deviations of the outcome variables at times $t-1$ and $t$, respectively [30]. Tracking coefficients $<0.3$ were considered weak, $0.3-0.6$ moderate, and $>0.6$ strong [11].

\section{Results}

A total of 758 (76\%), 472 (83\%) and 478 (84\%) children had valid accelerometry data at T1, T2 and T3, respectively (see Fig. 1). There were no significant differences in baseline characteristics (child age, child sex, child BMI, maternal age, and maternal education) between those who participated at either follow up and those who did not (data not shown). Mean accelerometer wear times were 11.5 $(\mathrm{SD}=1.2), 12.0(\mathrm{SD}=1.0)$ and $12.3(\mathrm{SD}=1.3)$ hours per day at T1, T2 and T3, respectively. Children had a mean age of 4.6 years $(\mathrm{SD}=0.7)$ at $\mathrm{T} 1,7.6$ years $(\mathrm{SD}=0.7)$ at $\mathrm{T} 2$, and 10.6 years $(\mathrm{SD}=0.7)$ at $\mathrm{T} 3$. At baseline, just over half ( $54 \%$ ) the children were boys and $55 \%$ of mothers were university educated.

\section{Changes in volume and accumulation patterns of physical activity and sedentary time}

Figures 2, 3 and 4 show the average total volumes of SED and physical activity intensities, bouts of SED, and 


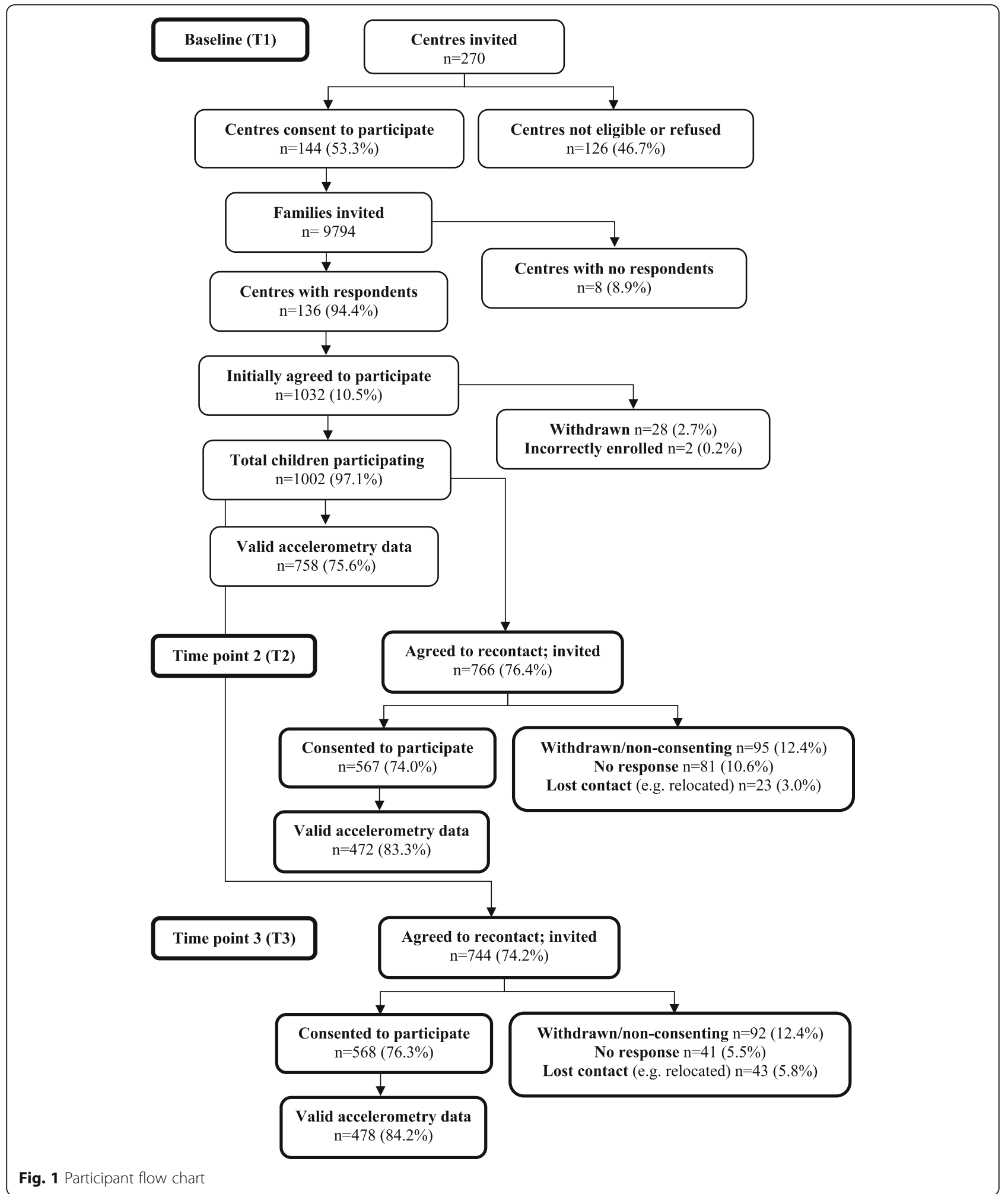

bouts of physical activity intensities, respectively, for boys and girls. The average number of breaks in SED over time for boys and girls is shown in Fig. 5. At all timepoints, girls engaged in significantly more SED and less MPA and VPA than boys (all $p<0.001$ ). There were no sex differences in average time spent in LPA until T3, where boys were more active than girls $(p=0.03)$. Coefficients for average change over time in each of the 
(a) Boys

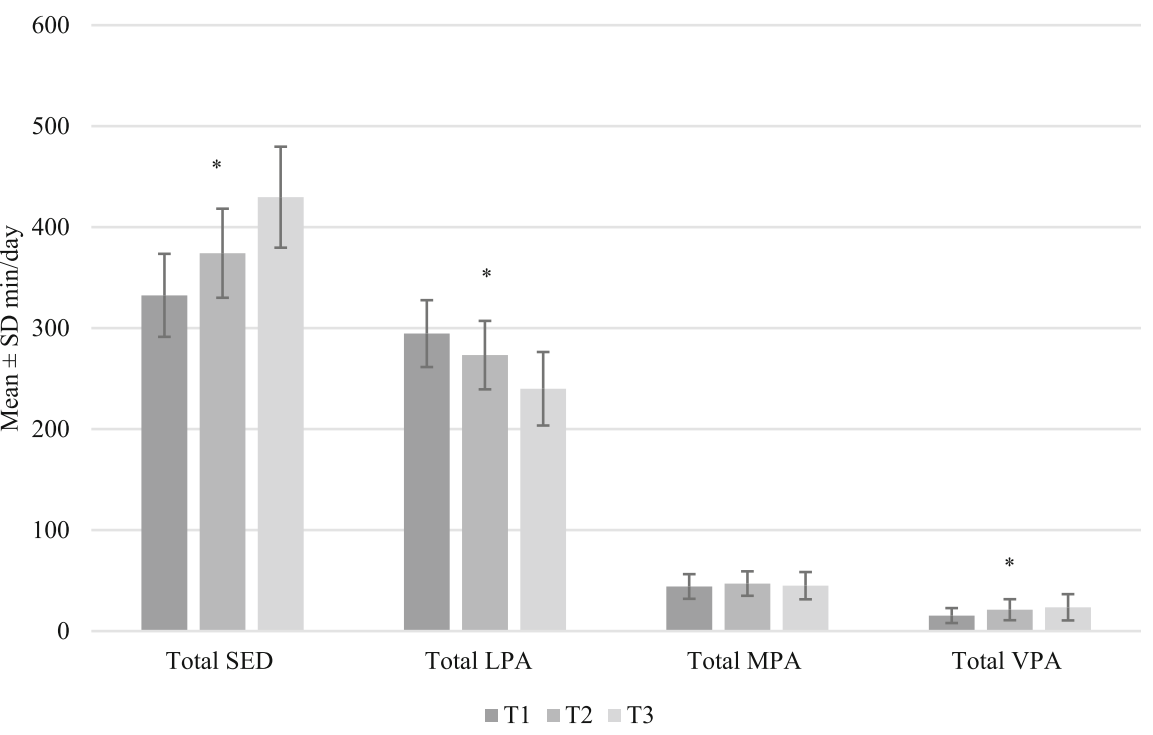

(b) Girls

$$
600
$$

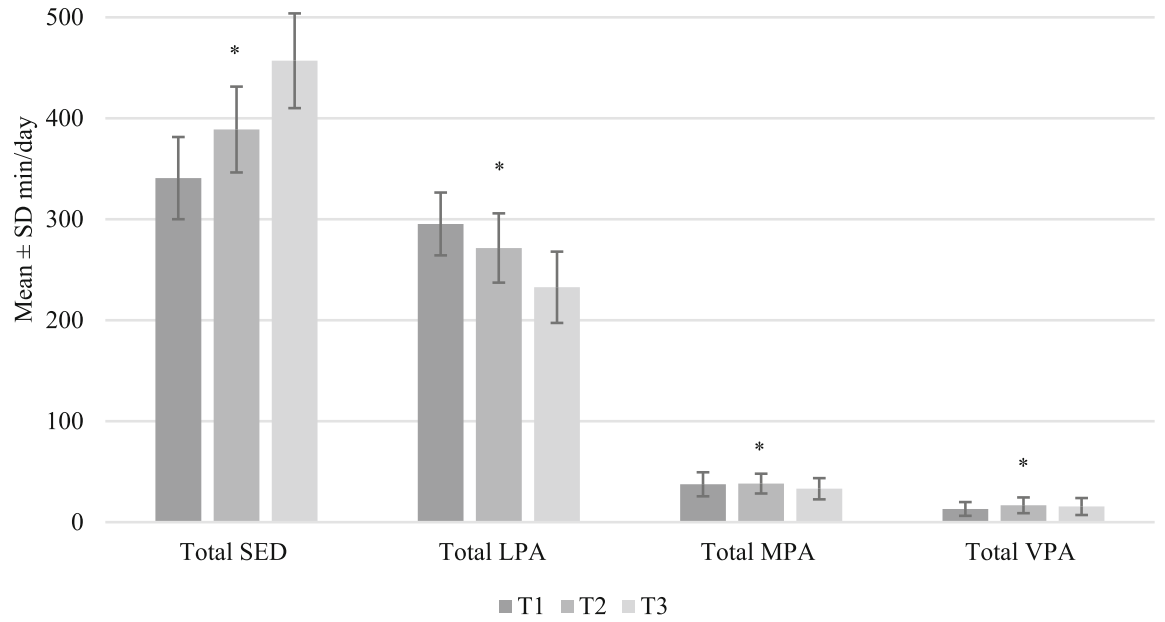

Fig. 2 Total volumes of sedentary time and physical activity from T1 to T3 for boys and girls* $p<0.001$ for change over time

total volume and pattern variables are shown in Table 1. For both boys and girls, total volume of SED, breaks in SED, and all bout lengths of SED increased over time. Conversely, total volume and bouts of LPA decreased over time for both sexes. There was a small decrease in total volume of MPA for girls only; however, time spent in $\geq 1$-min MPA bouts increased for both sexes over time. Total volume of VPA increased for both sexes, with time spent in $\geq 1$-min bouts of VPA increasing for boys only, and no change observed for time spent in $\geq 5$ min bouts for either boys or girls.
Tracking of volume and accumulation patterns of physical activity and sedentary time

Stability coefficients for each of the variables are shown in Table 2. For boys, there was moderate tracking of all volume and pattern variables except all SED bouts $\geq 15$ min, LPA bouts $\geq 5$-min and MPA bouts $\geq 1$-min (which all tracked weakly). For girls, there was strong tracking for total SED and SED bouts $\geq 1$-min. Moderate tracking was found for girls' total volume of LPA, MPA and VPA, SED bouts $\geq 5$ - min and $\geq 10-$ min SED bouts, LPA bouts $\geq 1$-min and MPA bouts $\geq 1$-min, and weak 
(a) Boys

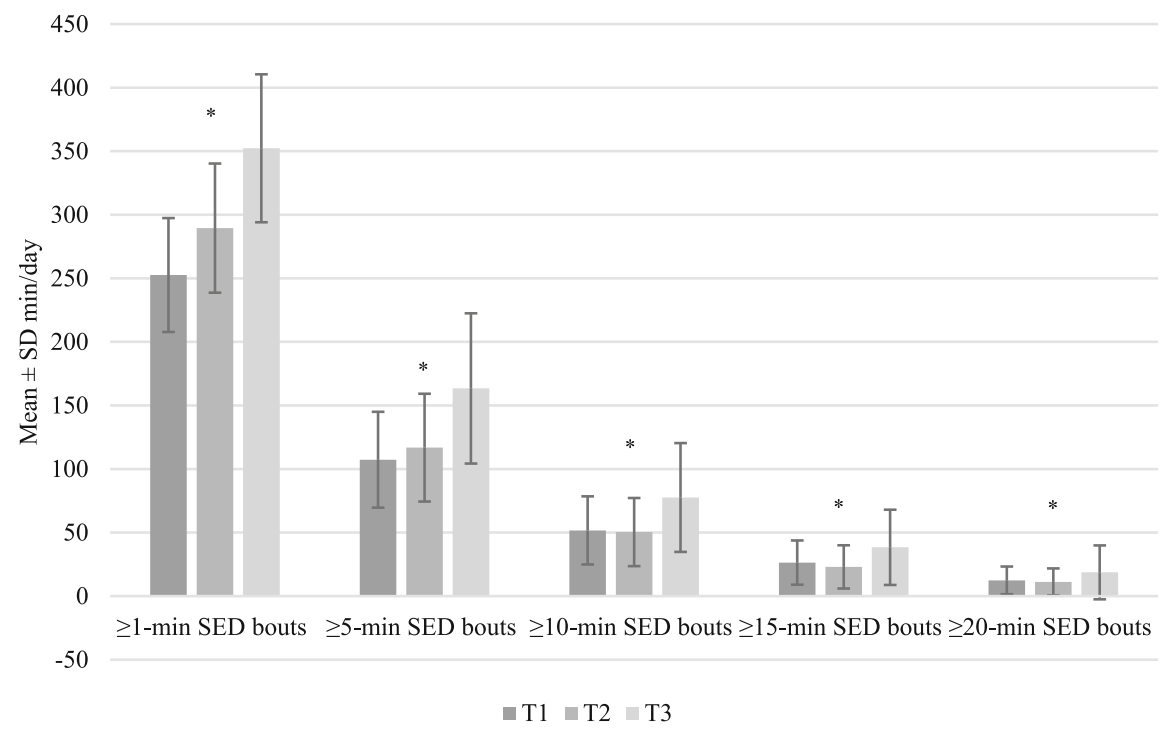

(b) Girls

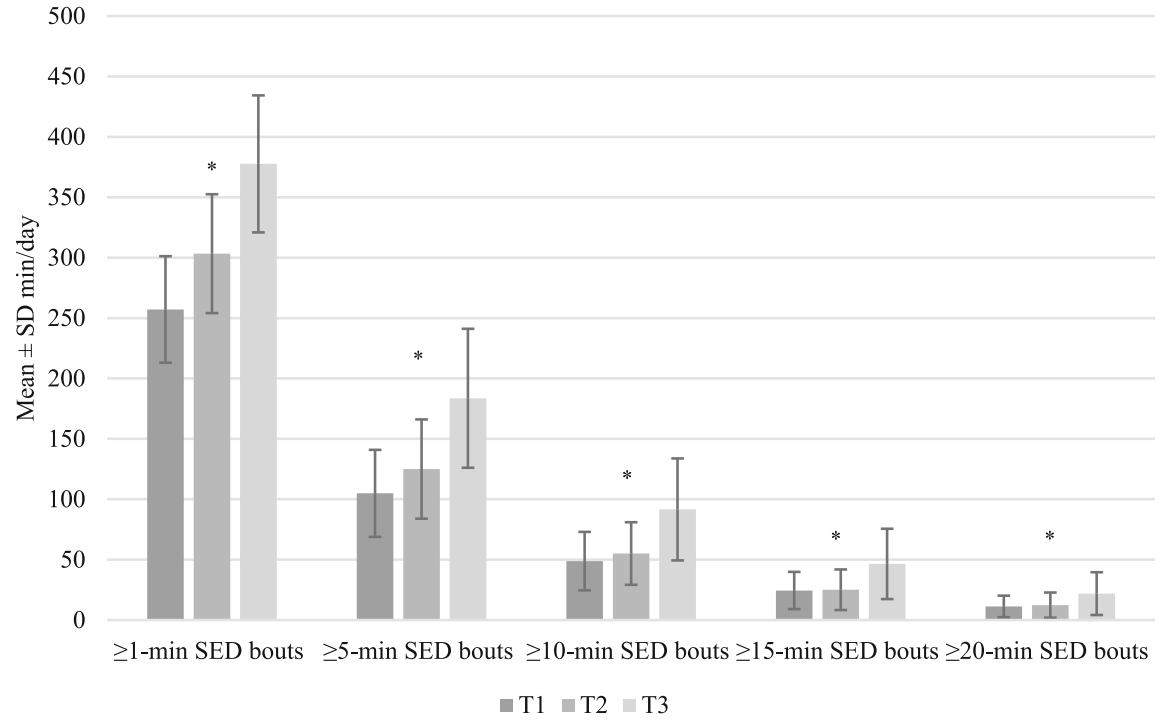

Fig. 3 Bouts of sedentary time from T1 to T3 for boys and girls* $p<0.001$ for change over time

tracking was found for girls' SED breaks, all SED bouts $\geq$ 15-min, LPA bouts $\geq 5$-min and all VPA bouts.

\section{Discussion}

This study is the first to our knowledge to investigate longitudinal changes and tracking of total volume and accumulation patterns of physical activity and SED across three timepoints from early to late childhood among boys and girls. Given increasing evidence of the importance of patterns of physical activity and SED for health $[3-5,31]$ and the need to identify opportunities to intervene, these findings are timely. We found that total volume and bouts of SED increased for both sexes across the three time points, while total volume and bouts of LPA decreased. Although we found a small decrease in total volume of MPA for girls only, time spent in $\geq 1$-min bouts of MPA increased and total volume of VPA increased for both sexes. Our findings are consistent with previous studies that have shown small increases in physical activity across 1-2 year periods in children aged 3-4 years at baseline [32-34]. Similarly, although Taylor et al. [35] observed a decline in MVPA 
(a) Boys

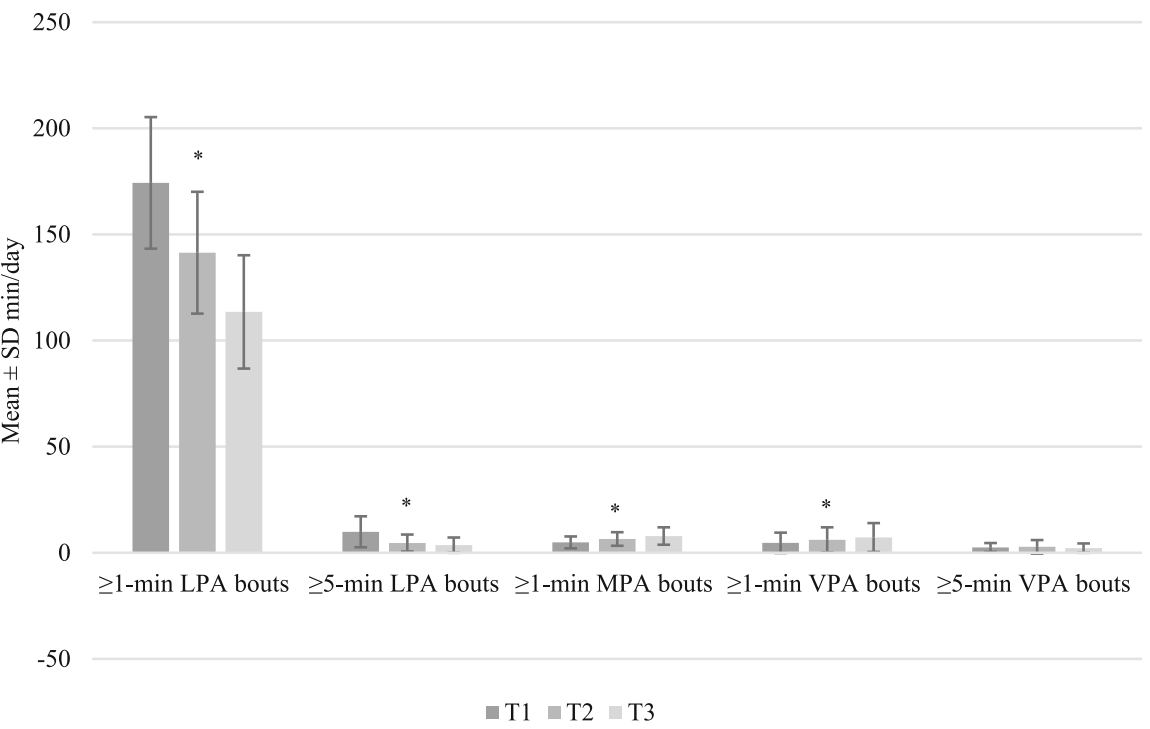

(b) Girls

$$
250
$$

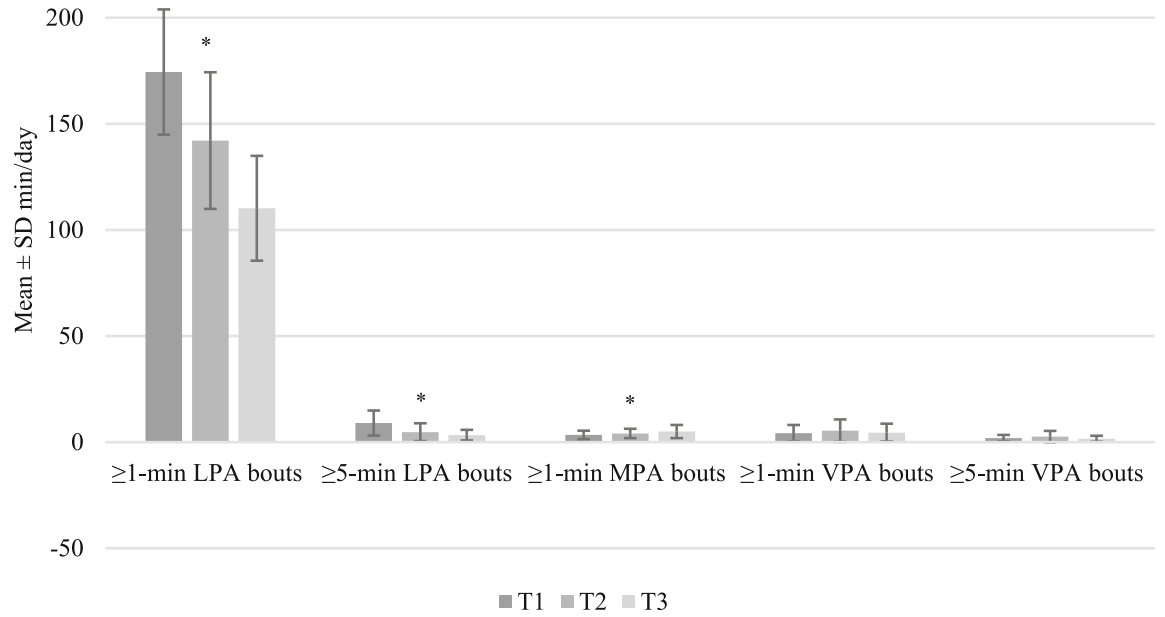

Fig. 4 Bouts of physical activity intensities from T1 to T3 for boys and girls* $p<0.001$ for change over time

from age 3 to 4 years, they found that it remained relatively consistent from age 4 to 7 years. They concluded that the increases in SED observed in their study predominantly occurred at the cost of activities undertaken at a light intensity.

Findings from this study highlight the need for interventions from an early age to focus on reducing SED by shifting children's movement along the activity spectrum. The amount of time spent in SED is potentially largely driven by screen time. Screens are ubiquitous and evidence suggests that fewer than $20 \%$ of preschool aged children meet recommendations for screen time [6]. Additionally, findings from Jones et al. [12] show that TV viewing is particularly stable during early childhood and from early to middle childhood, which may be driving the stability of overall SED. As such, a potential strategy for reducing SED may be to focus on reductions in sedentary screen time. Our findings also suggest that it is important to establish high levels of MVPA in early childhood, as children may then be more likely to maintain higher levels over time. However, young children are unlikely to be able to achieve 


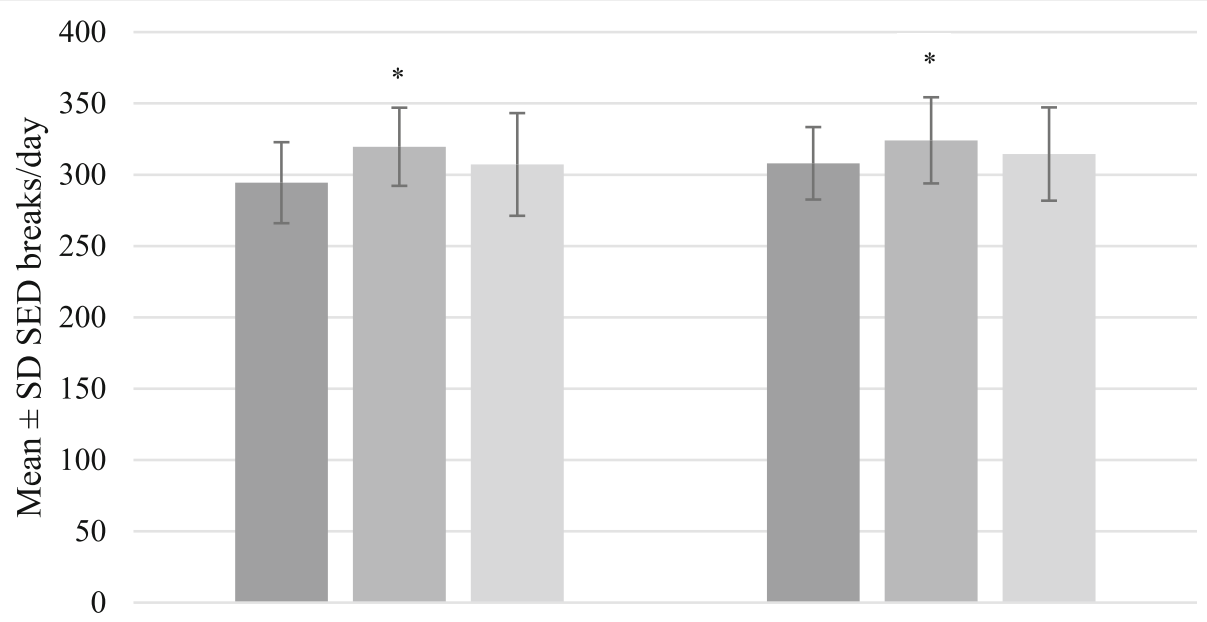

(a) Boys

(b) Girls

$\because \mathrm{T} 1 \quad \mathrm{~T} 2 \quad \mathrm{~T} 3$

Fig. 5 Breaks in sedentary time from T1 to T3 for boys and girls* $p<0.001$ for change over time

long bouts of MVPA from a physiological or cognitive perspective and tend to have limited tolerance for sustained MVPA [36]. As such, interventions should focus on reducing SED, maximising time spent in LPA, and promoting adequate levels of total MVPA accumulated in shorter bouts, to include appropriate rest breaks.

In terms of tracking, our findings suggest that total volume of SED, LPA, MPA and VPA are moderately to highly stable from early to later childhood for both boys and girls. However, tracking of the bouts variables was less consistent. Although there was moderate to strong tracking of shorter ( $\geq 1$ - to $\geq 10$-min) bouts of SED for both sexes, $\geq 15$ - and $\geq 20$-min bouts tracked weakly. This is largely consistent with findings from Carson et al. [15], who found moderate tracking of total SED and shorter SED bouts (1-4 and 5-9 min), but no tracking of $\geq 10$-min SED bouts among 3- to 5-year-olds over a 1 year period. These collective findings are likely because children accumulate SED differently as they get

Table 1 Average change from T1 to T3 in total volume and accumulation patterns of physical activity and sedentary time

\begin{tabular}{|c|c|c|c|}
\hline & \multicolumn{3}{|l|}{$\beta(95 \% \mathrm{Cl})^{1}$} \\
\hline & Boys & Girls & Total sample \\
\hline Total SED (min/day) & $48.2(44.8,51.7)$ & $57.8(54.1,61.4)$ & $52.7(50.1,55.2)$ \\
\hline Total LPA (min/day) & $-26.9(-29.6,-24.3)$ & $-31.2(-34.0,-28.4)$ & $-28.9(-30.8,-27.0)$ \\
\hline Total MPA (min/day) & $0.5(-0.4,1.5)$ & $-2.0(-2.9,-1.1)$ & $-0.7(-1.4,0.1)$ \\
\hline Total VPA (min/day) & $4.2(3.4,5.0)$ & $1.4(0.7,2.0)$ & $2.9(2.4,3.4)$ \\
\hline SED breaks (number/day) & $7.7(5.2,10.1)$ & $4.2(1.8,6.6)$ & $6.1(4.3,7.8)$ \\
\hline$\geq 1$-min SED bouts (min/day) & $49.0(45.1,52.9)$ & $59.7(55.5,63.9)$ & $54.0(51.1,56.9)$ \\
\hline$\geq 5$-min SED bouts (min/day) & $26.8(23.2,30.4)$ & $38.2(34.4,42.0)$ & $32.1(29.5,34.8)$ \\
\hline$\geq 10$-min SED bouts (min/day) & $12.0(9.5,14.5)$ & $20.4(17.8,23.1)$ & $15.9(14.0,17.7)$ \\
\hline$\geq 15$-min SED bouts (min/day) & $5.4(3.7,7.1)$ & $10.3(8.6,12.1)$ & $7.7(6.4,8.9)$ \\
\hline$\geq 20$-min SED bouts (min/day) & $2.9(1.7,4.2)$ & $5.2(4.0,6.4)$ & $4.0(3.1,4.8)$ \\
\hline$\geq 1$-min LPA bouts (min/day) & $-30.6(-32.8,-28.4)$ & $-32.6(-34.9,-30.3)$ & $-31.5(-33.1,-29.9)$ \\
\hline$\geq 5$-min LPA bouts (min/day) & $-3.3(-3.8,-2.9)$ & $-3.1(-3.5,-2.7)$ & $-3.2(-3.5,-2.9)$ \\
\hline$\geq 1$-min MPA bouts (min/day) & $1.5(1.2,1.7)$ & $0.8(0.6,1.0)$ & $1.2(1.0,1.4)$ \\
\hline$\geq 1$-min VPA bouts (min/day) & $1.3(0.8,1.7)$ & $0.2(-0.2,0.6)$ & $0.8(0.5,1.1)$ \\
\hline$\geq 5$-min VPA bouts (min/day) & $-0.1(-0.5,0.2)$ & $-0.1(-0.4,0.2)$ & $-0.1(-0.3,0.2)$ \\
\hline
\end{tabular}

Notes: ${ }^{1}$ Mixed models controlling for age at T1; boldface indicates $p<0.05$

Abbreviations: $C$ confidence interval; LPA light-intensity physical activity; MPA moderate-intensity physical activity; SED sedentary time; VPA vigorous intensity physical activity 
Table 2 Tracking coefficients for total volume and accumulation patterns of physical activity and sedentary time

\begin{tabular}{|c|c|c|c|}
\hline & \multicolumn{3}{|c|}{ Standardised tracking $(\beta)$ coefficients $^{1}$} \\
\hline & Boys & Girls & Total sample \\
\hline Total SED (min/day) & 0.49 & 0.64 & 0.56 \\
\hline Total LPA (min/day) & 0.52 & 0.58 & 0.54 \\
\hline Total MPA (min/day) & 0.35 & 0.56 & 0.52 \\
\hline Total VPA (min/day) & 0.54 & 0.60 & 0.58 \\
\hline SED breaks (number/day) & 0.32 & 0.28 & 0.32 \\
\hline$\geq 1$-min SED bouts (min/day) & 0.46 & 0.62 & 0.53 \\
\hline$\geq 5$-min SED bouts (min/day) & 0.39 & 0.47 & 0.41 \\
\hline$\geq 10-\min$ SED bouts (min/day) & 0.30 & 0.34 & 0.31 \\
\hline$\geq 15$-min SED bouts (min/day) & 0.24 & 0.23 & 0.24 \\
\hline$\geq 20$-min SED bouts (min/day) & 0.14 & 0.16 & 0.16 \\
\hline$\geq 1$-min LPA bouts (min/day) & 0.60 & 0.42 & 0.53 \\
\hline 25-min LPA bouts (min/day) & 0.14 & 0.03 & 0.05 \\
\hline$\geq 1$-min MPA bouts (min/day) & 0.22 & 0.33 & 0.35 \\
\hline$\geq 1$-min VPA bouts (min/day) & 0.41 & 0.26 & 0.35 \\
\hline$\geq 5$-min VPA bouts (min/day) & 0.40 & 0.28 & 0.43 \\
\hline
\end{tabular}

Notes: ${ }^{1}$ Tracking coefficients defined as weak $(<0.30)$, moderate $(0.30-0.60)$, and strong $(>0.60)$; boldface indicates $p<0.05$ for to non-standardised coefficients Abbreviations: LPA light-intensity physical activity; MPA moderate-intensity physical activity; SED sedentary time; VPA vigorous intensity physical activity

older, highlighted by the large increases in average time spent in longer bouts in our study.

In terms of the physical activity pattern variables, we found moderate to strong tracking of shorter bouts and weak tracking of longer bouts of LPA, and weak to moderate tracking of bouts of MPA $(\geq 1$ min). While VPA bouts of $\geq 1-\mathrm{min}$ and $\geq 5$-min tracked moderately for boys, we found weak tracking of these bouts for girls. It may be that engagement in longer and higher intensity bouts of physical activity is more variable as children age, given that they are more physically challenging to achieve. As children transition from preschool to primary school, there may also be fewer opportunities for them to engage in spontaneous physical activity. For girls specifically, it is likely that social norms increasingly influence their physical activity patterns as they age [37, 38]. The comparatively little amount of time spent in $\geq 5$ min LPA, $\geq 1$-min MPA, $\geq 1$-min VPA and $\geq 5$-min VPA bouts may also partly explain the weaker tracking than that of $\geq 1$-min LPA bouts. In addition, tracking coefficients for $\geq 5$-min bouts of LPA and VPA for girls were not statistically significant. This may be due to lack of power given the small numbers of children participating in bouts of this duration. For example, only around one quarter of girls included in analyses spent time in $\geq 5$-min bouts of VPA at each time point.

Contrary to previous research that has found that physical activity tends to track more strongly for boys than girls $[28,39]$, we found that while tracking was evident for both sexes, average total time in SED and all intensities of physical activity tended to be more stable for girls than boys. This, combined with the findings that SED was consistently higher, and MPA and VPA consistently lower for girls than boys over the three time points, suggests that girls may need additional support to reduce SED and engage in sufficient levels of MPA and VPA for health benefits. This underscores the need for physical activity interventions that target girls specifically, particularly as evidence consistently shows that girls are less active than boys [40-42]. Future research should aim to determine what factors influence girls' and boys' activity patterns, in order to design effective interventions.

A limitation of the current study is the loss to follow up. The HAPPY Study was initially designed as a crosssectional study, so follow-up samples were limited to those who had consented to be re-contacted. However, response rates among those contacted at both follow up time points were around $75 \%$. Strengths of the current study include the longitudinal design, which allowed the investigation of changes and tracking over three time points, and objective measures of sedentary time and physical activity. In addition, the examination of changes in and tracking of pattern variables is novel.

\section{Conclusions}

Children's activity patterns changed over time from preschool (3-5 years) to late primary school (9-11 years), with total volumes and short bouts of physical activity 
and SED tracking more strongly than longer bouts. Although MPA and VPA appeared to increase slightly, total volume and bouts of SED increased considerably more and, of concern, seemed to replace LPA. Future research should aim to determine what factors influence these changes in activity patterns so that effective interventions can be designed to ensure that children establish and maintain healthy physical activity and SED levels from a young age.

\section{Abbreviations}

LPA: Light-intensity physical activity; MPA: Moderate-intensity physical activity; MVPA: Moderate- to vigorous-intensity physical activity; PA: Physical activity; SED: Sedentary time; VPA: Vigorous-intensity physical activity

\section{Acknowledgements}

The authors are grateful to Simone Verswijveren for her assistance with bout analyses.

\section{Authors' contributions}

KLD conceptualized and designed the current study, carried out the analyses, drafted the initial manuscript, and reviewed and revised the manuscript. TH, AT, JS, AC, DPC, ADO and KDH conceptualized and designed the overall study, provided input into the data collection instruments, and critically reviewed the manuscript for important intellectual content. All authors approved the final manuscript as submitted and agree to be accountable for all aspects of the work.

\section{Funding}

HAPPY was funded by Deakin University and Australian Research Council Discovery Grants (DPI10101434 and DP140100554). KLD is supported by an Alfred Deakin Postdoctoral Research Fellowship. TH was supported by a Deakin University APA PhD Scholarship and a National Health and Medical Research Council Early Career Fellowship (APP1070571) during the study. $\mathrm{KDH}$ is supported by an Australian Research Council Future Fellowship (FT130100637) and an Honorary National Heart Foundation of Australia Future Leader Fellowship. JS is supported by a National Health and Medical Research Council Leadership Level 2 Fellowship (APP1176885). AT was supported by a National Heart Foundation Future Leader Fellowship (Award 100046) during the study. The funders played no role in the design of this study during its execution, analyses, interpretation of the data, or decision to submit results.

\section{Availability of data and materials}

The datasets analysed for the current study are not publicly available due to ethical restrictions related to the consent given by participants at the time of study commencement. An ethically compliant dataset may be made available by the corresponding author on reasonable request and upon approval by the Deakin University Human Research Ethics Committee.

\section{Declarations}

Ethics approval and consent to participate

Approval to conduct the HAPPY Study was granted by Deakin University's Human Research Ethics Committee (EC291-2007), Department of Education and Early Childhood Development (2011_001008), and Catholic Education Melbourne (GE11/0009). Participating parents provided written informed consent for themselves and their children to take part.

\section{Consent for publication}

Not applicable.

\section{Competing interests}

The authors declare that they have no competing interests.

\section{Author details}

${ }^{1}$ Institute for Physical Activity and Nutrition (IPAN), School of Exercise and Nutrition Sciences, Deakin University, Geelong, Australia. ${ }^{2}$ Mary MacKillop Institute for Health Research, Australian Catholic University, Melbourne, Australia. ${ }^{3}$ Early Start, Faculty of Social Sciences, Illawarra Health and Medical Research Institute, University of Wollongong, Wollongong, Australia.

Received: 30 August 2020 Accepted: 3 March 2021

Published online: 17 March 2021

\section{References}

1. Poitras VJ, et al. Systematic review of the relationships between sedentary behaviour and health indicators in the early years ( $0-4$ years). BMC Public Health. 2017;17(Suppl 5):868.

2. Carson $\mathrm{V}$, et al. Systematic review of the relationships between physical activity and health indicators in the early years ( $0-4$ years). BMC Public Health. 2017;17(Suppl 5):854.

3. Saunders TJ, et al. Associations of sedentary behavior, sedentary bouts and breaks in sedentary time with cardiometabolic risk in children with a family history of obesity. PLOS ONE. 2013;8(11):e79143.

4. Cliff DP, et al. Volumes and bouts of sedentary behavior and physical activity: associations with cardiometabolic health in obese children. Obesity. 2014;22(5):E112-E8.

5. Fletcher EA, et al. Effects of breaking up sitting on adolescents' postprandial glucose after consuming meals varying in energy: a cross-over randomised trial. J Sci Med Sport. 2018;21(3):280-5.

6. Cliff DP, et al. Adherence to 24-Hour Movement Guidelines for the Early Years and associations with social-cognitive development among Australian preschool children. BMC Public Health. 2017;17(5):857.

7. Hnatiuk JA, et al. Changes in volume and bouts of physical activity and sedentary time across early childhood: a longitudinal study. Int J Behav Nutr Phys Act. 2019;16(1):42.

8. Hinkley T, et al. Prospective associations with physiological, psychosocial and educational outcomes of meeting Australian 24-Hour Movement Guidelines for the Early Years. International Journal of Behavioral Nutrition Physical Activity. 2020;17(1):36.

9. Cooper AR, et al. Objectively measured physical activity and sedentary time in youth: the International children's accelerometry database (ICAD). Int J Behav Nutr Phys Act. 2015;12:113.

10. Bloom BS. Stability and Change in Human Characteristics. New York: John Wiley \& Sons, Inc; 1964.

11. Malina RM. Tracking of physical activity and physical fitness across the lifespan. Res Q Exerc Sport. 1996;67(3):48-57.

12. Jones RA, et al. Tracking physical activity and sedentary behavior in childhood: a systematic review. Am J Prev Med. 2013;44(6):651-8.

13. Carson $V$, et al. Physical activity and sedentary behavior across three timepoints and associations with social skills in early childhood. BMC Public Health. 2019;19(1):27-

14. Caldwell HA, et al. Tracking of physical activity and fitness during the early years. Appl Physiol Nutr Metab. 2016;41(5):504-10.

15. Carson V, et al. Longitudinal levels and bouts of objectively measured sedentary time among young Australian children in the HAPPY study. Journal of Science Medicine in Sport. 2016;19(3):232-6.

16. Abbott $\mathrm{G}$, et al. Cross-sectional and Longitudinal Associations Between Parents' and Preschoolers' Physical Activity and Television Viewing: The HAPPY Study. J Phys Act Health. 2016;13(3):269-74.

17. Cliff DP, et al. Methodological considerations in using accelerometers to assess habitual physical activity in children aged $0-5$ years. Journal of Science Medicine in Sport. 2009;12(5):557-67.

18. Sirard JR, et al. Calibration and evaluation of an objective measure of physical activity in preschool children. Journal of Physical Activity Health. 2005;2(3):345-57.

19. Cain KL, et al. Using accelerometers in youth physical activity studies: a review of methods. J Phys Act Health. 2013;10(3):437-50.

20. Hinkley $\mathrm{T}$, et al. Assessing volume of accelerometry data for reliability in preschool children. Med Sci Sports Exerc. 2012;44(12):2436-41.

21. Mattocks $C$, et al. Use of accelerometers in a large field-based study of children: protocols, design issues, and effects on precision. J Phys Act Health. 2008;5(Suppl 1):98-111.

22. Evenson KR, et al. Calibration of two objective measures of physical activity for children. J Sports Sci. 2008;26(14):1557-65. 
23. Janssen X, et al. Predictive Validity and Classification Accuracy of ActiGraph Energy Expenditure Equations and Cut-Points in Young Children. PLoS One. 2013;8(11):e79124.

24. Trost SG, et al. Comparison of Accelerometer Cut Points for Predicting Activity Intensity in Youth. Medicine \& Science in Sports \& Exercise 2011;43(7).

25. Aadland $\mathrm{E}$, et al. Associations of volumes and patterns of physical activity with metabolic health in children: A multivariate pattern analysis approach. Prev Med. 2018;115:12-8.

26. Gabel $\mathrm{L}$, et al. Associations of sedentary time patterns and TV viewing time with inflammatory and endothelial function biomarkers in children. Pediatric obesity. 2016;11(3):194-201.

27. Willett $W$, et al. Total energy intake: implications for epidemiologic analyses. Am J Epidemiol. 1986;124(1):17-27.

28. Carver A, et al. How is active transport associated with children's and adolescents' physical activity over time? Int J Behav Nutr Phys Act. 201 1:8:126.

29. Pearson N, et al. Tracking of children's body-mass index, television viewing and dietary intake over five-years. Prev Med. 2011;53(4-5):268-70.

30. Twisk JWR. Applied longitudinal data analyses for epidemiology: A practical guide. Cambridge: University Press; 2003.

31. Verswijveren SJJM, et al. Associations between activity patterns and cardiometabolic risk factors in children and adolescents: A systematic review. PLoS One. 2018:13(8):e0201947-e.

32. Kelly LA, et al. Tracking physical activity and sedentary behavior in young children. Pediatr Exerc Sci. 2007;19(1):51-60.

33. Jackson DM, et al. Objectively Measured Physical Activity in a Representative Sample of 3- to 4-Year-Old Children. Obes Res. 2003;11(3):420-5.

34. McKee DP, et al. Seasonal and annual variation in young children's physical activity. Med Sci Sports Exerc. 2012;44(7):1318-24.

35. Taylor RW, et al. Changes in Physical Activity over Time in Young Children: A Longitudinal Study Using Accelerometers. PLOS ONE. 2013;8(11):e81567.

36. Bailey RC, et al. The level and tempo of children's physical activities: an observational study. Med Sci sports Exerc. 1995;27(7):1033-41.

37. Vu MB, et al. Listening to girls and boys talk about girls' physical activity behaviors. Health Educ Behav. 2006;33(1):81-96.

38. Allender $\mathrm{S}$, et al. Understanding participation in sport and physical activity among children and adults: a review of qualitative studies. Health Educ Res. 2006;21(6):826-35.

39. Potter M, et al. Behavior Tracking and 3-Year Longitudinal Associations Between Physical Activity, Screen Time, and Fitness Among Young Children Pediatr Exerc Sci. 2018;30(1):132-41.

40. Hallal PC, et al. Global physical activity levels: surveillance progress, pitfalls, and prospects. The Lancet. 2012;380(9838):247-57.

41. Trost SG, et al. Age and gender differences in objectively measured physical activity in youth. Med Sci Sports Exerc. 2002;34(2):350-5.

42. Pearce MS, et al. Early predictors of objectively measured physical activity and sedentary behaviour in 8-10 year old children: the Gateshead Millennium Study. PLOS ONE. 2012;7(6):e37975.

\section{Publisher's Note}

Springer Nature remains neutral with regard to jurisdictional claims in published maps and institutional affiliations.

Ready to submit your research? Choose BMC and benefit from:

- fast, convenient online submission

- thorough peer review by experienced researchers in your field

- rapid publication on acceptance

- support for research data, including large and complex data types

- gold Open Access which fosters wider collaboration and increased citations

- maximum visibility for your research: over $100 \mathrm{M}$ website views per year

At BMC, research is always in progress.

Learn more biomedcentral.com/submissions 